\title{
La lexicografía del español de América y un nuevo diccionario de americanismos
}

\author{
Günther Haensch \\ Universidad de Augsburgo
}

Antes de emitir un juicio sobre el Breve Diccionario Ejemplificado de Americanismos de Brian Steel (Arco/Libros, Madrid 1999, 288 pp., de ahora en adelante citado como Steel 1999), me parece oportuno hacer algunas observaciones generales sobre la lexicografía del español de América para poder apreciar los méritos de la obra y, al mismo tiempo, explicar sus posibles limitaciones. Aprovecho la oportunidad para hacer unos comentarios generales sobre los diccionarios del español de América con el fin de ilustrar la problemática de su valoración por parte de los hispanistas y para recordarnos que hay que obrar con mucha prudencia para hacer justicia a los repertorios lexicógrafos del español de América.

\section{LA "NUEVA LEXICOGRAFÍA” HISPANOAMERICANA}

Tras haberme dedicado 25 años a la lexicografía y a la metalexicografía del español de América, especialmente como co-director del "Proyecto de Augsburgo: Nuevo Diccionario de Americanismos"1 (conjuntamente con Reinhold Werner, su actual director), he llegado a las siguientes conclusiones:

1 Sobre este proyecto. Vid. Haensch (1983), id. (1984 a), id. (1984 b), id. (1990 a); id. (1997, reimp. 1999), pp. 227-228; Haensch-Werner (1982 a), id. (1982 b), id. (1992); Werner (1978). Más bibliografia da Haensch (1997), p. 227, nota 282. 
1. El alcance de la profunda renovación de la lexicografía del español de América, que se produjo en los últimos 25 años con la publicación de los nuevos diccionarios contrastivos del español de Chile ${ }^{2}$, de Colombia $^{3}$, de Venezuela ${ }^{4}$, de Argentina ${ }^{5}$ y de Uruguay $^{6}$, y de los diccionarios del proyecto Diccionario del Español de México (DEM) ${ }^{7}$, sólo ha sido valorado por pocos especialistas, entre ellos Manuel Seco, quien habló de la "nueva lexicografía del español de América"". Al referirnos a ésta, no queremos, de ninguna manera, mermar los méritos de los anteriores diccionarios del español de América, cuyas virtudes y deficiencias, éstas a veces muy graves, hemos tratado mi colega Werner y yo en una serie de publicaciones'; pero es innegable que con la "nueva lexicografía hispanoamericana" se dio un gran paso adelante, especialmente en cuanto al método de la descripción lexicográfica. El que este hecho, por lo general, no se haya juzgado en su justo valor en el mundo de la filología y lingüística hispánicas se debe, en mi opinión, a dos causas: por un lado, a la desconcertante complejidad del léxico del español hablado en América; por otro lado, a la mayor medi$\mathrm{da}$, a un conocimiento sólo parcial del peculiar mundo de la lexicografía, su metodología y sus servidumbres que tiene la mayoría de los hispanistas que no se hayan dedicado a trabajos lexicográficos o metalexicográficos.

2. Algunos colegas han criticado o por lo menos lamentado, el he-

2 Morales-Quiroz (1983).

3 Haensch-Werner (eds.) (1993 a).

4 Tejera (1993) y Núñez-Pérez (1994).

5 Haensch-Werner (eds.) (1993 b).

6 Haensch-Werner (eds.) (1993 c).

7 Lara (1982/1993), Lara (1986) y Lara (1996).

8 Seco (1995). Glessgen (1998) y Lüdtke (1997) destacan en sus reseñas el carácter innovativo de los diccionarios del "Proyecto de Augsburgo".

9 Vid. Haensch (1978); id. (1980); id. (1984 c); id. (1986); id. (1987); id. (1988); id. (1989); id. (1991); Haensch/Werner (1970); Werner (1979); Werner (1984); Werner (1991); Werner (1994); Werner (1996). 
cho de que en los diccionarios del "Proyecto de Augsburgo" no se hayan indicado etimologías. Esto, en primer lugar, no es necesario ni usual en un diccionario descriptivo que tiene otra finalidad; en segundo lugar, me gustaría que alguien me explicara dónde hay especialistas en docenas de lenguas amerindias, que conozcan al mismo tiempo la evolución histórica de éstas y la gramática histórica del español, para investigar los casos de las etimologías amerindias no resueltos hasta hoy; todo ello ante la falta de un número suficiente de estudios de documentos y relatos desde el Descubrimiento hasta el siglo XIX. Lo que algunos diccionarios del español de América ofrecen son indicaciones del tipo "voz procedente del náhuatl" o "palabra de origen cumanagoto". Empero, ¿qué fiabilidad puede tener una vaga indicación etimológica sobre una palabra sin dar su étimo en la lengua indígena de origen, su significado en ésta y los posibles cambios fonéticos y semánticos que sufrió la voz una vez incorporada en el léxico español? Claro está que muchas etimologías se conocen ya debido a su primera ocurrencia en un texto (crónica, relato) referido a una comunidad indígena concreta, pero, aún mucho más, debido a la meritoria labor de lingüistas que son, al mismo tiempo, conocedores de una lengua indígena y del español. Es cierto que el diccionario etimológico de CorominasPascual (1980-1991) ofrece muchas etimologías amerindias; hay, sin embargo, en una serie de variantes del español de América, muchas palabras cuya etimología es desconocida o, por lo menos, controvertida. Son palabras que, en su mayoría, deben proceder de una lengua indígena y, en el peor de los casos, de una de las muchas ya extinguidas. Recordemos, de paso, que en Colombia existían todavía unas 100 lenguas indígenas en $1968^{10}$, de las que hoy quedan apenas unas docenas, con un número reducido de hablantes.

3. La inacabable y, en el fondo, estéril discusión sobre lo que es un americanismo, argentinismo, colombianismo, etc., lo cual es cuestión de puro nominalismo, ha dado lugar a interpretaciones arbitrarias y erróneas. Así, por ejemplo, Klaus Zimmermann, al que debemos valio-

10 Vid. Caudmont (1968), p. 1196. 
sos estudios sobre el español de América, afirma, refiriéndose al Nuevo Diccionario de Colombianismos (NDC), lo siguiente: "La concepción del diccionario se basa, por un lado, en una definición estrictamente contrastiva de [la noción de] colombianismo, y, por ende, no registra las voces que, si bien tienen su origen histórico en Colombia, ya se usan hoy en día también en otra parte; por otro lado, el criterio para determinar lo específicamente colombiano es el español europeo. La consecuencia de esto es que un número bastante elevado de palabras usuales en la vecina Venezuela o en otros países hispanoamericanos se clasifican erróneamente como colombianismos"11. Aquí, el colega hispanista se equivoca. Como se expone en la Presentación, el Prólogo y la Introducción del Nuevo Diccionario de Colombianismos, éste registra unidades léxicas usuales en Colombia que presentan una diferencia de uso frente al español peninsular o no existen en éste. ¿De dónde saca el Sr. Zimmermann que las unidades léxicas registradas en el NDC son exclusivas de Colombia o pretenden serlo? Los directores y redactores del diccionario, que sabían desde el inicio de los trabajos perfectamente que muchas de las unidades léxicas registradas en el NDC se usan en otras áreas hispanoamericanas, no se han referido en ninguna parte en el NDC al exclusivismo que nuestro colega atribuye al término de "colombianismo". Los diccionarios del "Proyecto de Augsburgo" que serán publicados de ahora en adelante por la Editorial Gredos (Madrid) se llamarán "Diccionario del español de Cuba", "Diccionario del español de Bolivia", etc., lo que tampoco significa que las unidades léxicas registradas en éste no se usen también en otros países hispanoamericanos, pero a lo mejor este cambio de nombre servirá para que no se den nuevas interpretaciones exclusivistas del léxico de un país hispanoamericano frente al de otros.

11 Zimmermann (1997). La traducción de la cita es nuestra. 


\section{EL DICCIONARIO DE STEEL (1999)}

De lo expuesto hasta ahora se puede colegir que no es nada fácil emitir un juicio equitativo sobre cualquier inventario de unidades léxicas del español de América. Lo intentaremos con la obra de Brian Steel.

\section{MACROESTRUCTURA Y MICROESTRUCTURA DE LA OBRA}

En primer lugar, cabe preguntarse qué finalidad pretende cumplir un diccionario. En cuanto a la obra que comentamos, el autor lo dice claramente: "Este Breve Diccionario Ejemplificado [...] sólo ofrece una selección de americanismos a base de los ejemplos de uso que se han encontrado en la calle, la literatura, el periodismo, el cine, y en los diccionarios nacionales, sobre todo en un número creciente de obras de gran solvencia que hace unos diez años empezaron a aparecer tras muchos años de intensa labor por equipos de investigadores lexicológicamente progresistas" (p. X). No se trata, pues, de un diccionario general ni de un diccionario básico elaborado según criterios de frecuencia, sino de un inventario de unidades léxicas usuales en el español de América y documentadas de una $u$ otra forma. El ofrecer ejemplos documentados de uso es el principal mérito del diccionario. En la primera parte de la obra, dedicada a los americanismos, stricto sensu, se dan, en cada entrada después del lema (en negrita), indicaciones gramaticales y luego se señala donde se usa la unidad léxica americana en cuestión, seguido -casi siempre- de su equivalente en español peninsular. Siguen la cita o las citas con indicación de su fuente y, al final de cada artículo, se da el equivalente de la unidad léxica descrita en inglés, lo cual, entre otras cosas, permite precisar el significado del lema. En el caso de unidades léxicas procedentes de otras lenguas, bien sean europeas, o bien amerindias, se señala, después de las indicaciones gramaticales, la lengua de origen de éstas mediante siglas sin más explicaciones.

En un apéndice se ofrecen "Otros americanismos morfológicos y sintácticos" (pp. 243-248). En la segunda parte se registran "Americanismos de frecuencia", es decir, unidades léxicas que se usan tanto en España como en uno o varios de los países hispanoamericanos, pero con distinta frecuencia. En la Parte III se da, siempre con aval de citas 
textuales, un inventario de "Palabras de origen latinoamericano que ahora son de uso general en español" (pp. 267-276). Al final figura una bibliografía. En la primera parte de ésta se indican las fuentes lexicográficas que aprovechó el autor; en la segunda parte se ofrece el listado de las fuentes escritas de donde proceden los ejemplos citados.

\section{FUENTES DEL DICCIONARIO}

En cuanto a los diccionarios y otros inventarios lexicográficos aprovechados por el autor, la selección de éstos parece adecuada. Sin embargo, hubiera sido preferible prescindir de algunos de ellos por su poca fiabilidad. Me refiero a las obras siguientes: Schwauss (1977) (bilingüe español americano-alemán), Arias de la Cruz (monolingüe) $(1980)^{12}$ y Americanismos (1983) (monolingüe y enciclopédico). Esta última obra no es otra cosa que un extracto de un diccionario enciclopédico, Gran Sopena (1980), publicado por la misma editorial, con el agravante de que el significado de las palabras está mejor explicado y la extensión geográfica mejor indicada en el diccionario general que en Americanismos (1983). Algunas de las entradas aparecen ya en un diccionario enciclopédico anterior de la misma editorial (Diccionario Sopena 1978) con las mismas definiciones. Sabido es que Morínigo (1969 y 1996) y Neves (1973) registran, entre otras cosas, bastantes peninsularismos.

\section{LIMITACIONES DEL DICCIONARIO}

La aplicación de un método lexicográfico basado esencialmente en la documentación es valiosa porque aporta pruebas del uso real y actual de las unidades léxicas registradas, pero naturalmente, trae consigo ciertas limitaciones inherentes a este método, que, de ningún modo, se pueden considerar como defectos.

12 Hay que señalar que la Editorial Everest publicó en 1997 otro diccionario de americanismos cuya selección del léxico y cuyas definiciones coinciden, en parte, aunque con cambios de redacción, con Arias de la Cruz (1980): Everest. Diccionario práctico de americanismos. León 1997, pp. 238. 
1. La primera de estas limitaciones es la selección del léxico. Esta es, lo que se llama en inglés 'random harvest', "cosecha del azar", aunque hay que reconocer que la mayoría de las unidades léxicas registradas pertenecen al léxico usual ${ }^{13}$.

Debido al criterio de selección de la obra, se echan de menos algunas palabras de uso frecuente en el español de América; como: cartón corrugado $\mathrm{Am}$ 'cartón ondulado'; contador $m \mathrm{Am}$ (sin las Antillas) 'contable'; durmiente $m \mathrm{Am}$ 'traviesa en la vía del tren' (AmMer, Am-

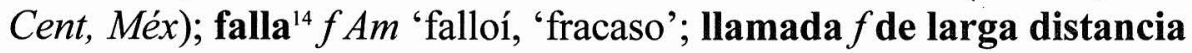
$A m$ 'conferencia interurbana o interprovincial'; picaflor $m$ 'colibrí' ( $A m M e r, C R, N i c, R D$ ); riel $m A m$ 'raíl de una vía férrea'; tren $m$ de carga $\mathrm{Am}$ 'tren de mercancías' (coloquial: el mercancías); video $\mathrm{m} \mathrm{Am}$ ( $¿$ a excepción de $P R$ ?) corresponde a vídeo $m$ en España.

2. La indicación de que una unidad léxica se usa un país determinado no tiene forzosamente un valor absoluto. La palabra registrada puede ser un regionalismo, a veces menos usual que un sinónimo de éste que no aparece en el diccionario o, aparece sin más explicaciones. Esto ocurre con palabras como catire 'rubio' que lleva las marcas $\mathrm{Col}, \mathrm{Cu}$ ba, Venezuela. En Colombia, sin embargo, la voz más usual es mono 'rubio'; catire se usa en las regiones fronterizas con Venezuela. Steel 1999 registra también mono 'rubio' $(\mathrm{Col})$, pero la diferencia en cuanto al uso de las dos palabras no se hace patente. Recámara 'dormitorio' que Steel registra con las marcas AmCent, Méx y Col se usa en Colombia sólo a nivel regional (en 5 departamentos).

13 De ahora en adelante usaremos las siguientes siglas para referirnos a los países hispanoamericanos: $\mathrm{Am}$ se usa en toda Hispanoamérica; $\mathrm{AmCent}=$ América Central; $A m M e r=$ América del Sur; $\mathrm{Arg}=$ Argentina; $B o l=$ Bolivia; $\mathrm{Col}=$ Colombia $C R=$ Costa Rica; $E c=$ Ecuador; Guat $=$ Guatemala; Hond $=$ Honduras; Méx $=$ Méjico; Nic $=$ Nicaragua Pan $=$ Panamá; $P a r=$ Paraguay; $P e=$ Perú; $P R=$ Puerto Rico; $R D=$ República Dominicana; $R P l=$ Río de la Plata; $S a l v=$ Salvador; $U r=$ Uruguay; $V e n=$ Venezuela. Usaremos las mismas siglas al referirnos a las marcas geográficas que da Steel (1999) (aunque en esta obra se usan otras siglas).

14 Steel 1999 registra la voz falla entre los usuales en España y América, pero nos parece que lo usual en España es fallo y en América falla $f$. 
3. El método de citas aplicado por el autor impide naturalmente que se consignen otras acepciones de una palabra registrada en el diccionario, a veces más frecuentes. Esto ocurre, por ejemplo, con "almorzar", en Steel 1999 'desayunar tarde' (en Méx.). La voz significa prácticamente en toda Hispanoamérica 'tomar la comida del mediodía' (ver, más abajo, nuestras observaciones sobre 'almuerzo'); “cajón $m$ 'caja de madera' (para el embalaje)" se usa en Arg., Ur., Par., Chile, Bol., Col., Ven., Pan., Hond., Salv., Méx., RD, en Steel 1999 se registra sólo con la acepción 'cañada larga' (Arg., Chile); "consignar $v t$ " en Col. 'ingresar dinero en una cuenta bancaria'; "carajo $m$ " significa en Colombia también 'individuo despreciable' (NDC); "chicote $m$ " tiene en Colombia también el significado de "colilla (del cigarrillo)'; "cocuyo $m$ " el de 'luz de posición de un automóvil' (NDC); "coquero $m$ " tiene hoy día en Colombia, además de las acepciones indicadas, la de "traficante de cocaína' (NDC); "joto m", en Steel 1999 'marica', significa en Colombia 'hato', 'conjunto de cosas atadas' (NDC); la palabra representada en el artículo "alzado $\mathrm{nm} \mathrm{Cuba}$ 'pequeño estante" es mucho más frecuente, en una serie de países hispanoamericanos, con la acepción 'rebelde', 'guerrillero'; para "vaina $n f f a m$ " con la marca Col. y Ven., el diccionario registra el equivalente 'chisme', que es correcto, pero la voz se usa más en Col. para referirse a un hecho o asunto cualquiera, por ejemplo '¿qué, es la vaina?', y significa, sobre todo, 'contrariedad', 'problema', como en el español de Argentina: “iqué macana!”.

\section{INCONSECUENCIAS DE MÉTODO}

Algunos puntos flacos de la obra se deben a falta de consecuencia en la aplicación del método adoptado por el autor.

1. El diccionario da, en muchas entradas, marcas geográficas que no están apoyadas todas ellas por una cita, por ej. "bañadera 1. $n f$ [Chile, $\mathbf{R p l}$ ] = bañera $\|$ Se sumergió en la bañadera semillana. (M. Puig, A., 1970, 54) bath; bathtub [...]".

Esta información seguramente es valiosa para el usuario, pero infringe el principio básico de los ejemplos documentados porque hay sólo documentación para Argentina (A.) y no para Chile, Paraguay y 
Uruguay. En realidad, el autor ha aplicado -en muchos artículos- un método mixto: ejemplos documentados para uno o dos países, completando la enumeración de países donde se usa la voz en cuestión mediante la consulta de diccionarios. Es una solución de compromiso. $\mathrm{Si}$ se aplicase este método consecuentemente, habría que añadir muchas marcas geográficas para informar al usuario que la voz se usa también en otros países. Más adelante intentaré completar la información de la obra con materiales que yo mismo he recogido.

2. Al usar el diccionario, resulta a veces difícil o imposible enterarse de la localización geográfica de los americanismos. En el artículo para "luz", "1. luz alta $n f=$ luz de carretera fullbeam (lights). 2 . luz baja $n f=$ luz de cruce dipped headlights" no se da ninguna indicación sobre la extensión de uso de la voz. Concluir de allí que se usan en toda Hispanoamérica, sería un error, puesto que en Uruguay, Cuba y Puerto Rico, por ejemplo, corresponde a 'luz baja': luz $f$ corta. A 'luz alta' corresponden en $U r$ y $P R$ : luz $f$ larga. En el artículo para "ahuevado", que significa en España 'atontado', se remite sólo a la obra de Hediger (1977) (difícilmente accesible para el usuario común y corriente) y, por lo tanto, el usuario del diccionario no se entera de la localización geográfica de la palabra.

3. Lo que es más grave, en algunos casos no se indica ninguna fuente, por ej. en la entrada abombado,-a adj y nmf [Rpl] 'majadero'; en aerosilla $n f$ (rioplatense) 'telesilla' y en dúrex 'scotch'15, 'cinta adhesiva', etc, etc.

15 Scotch es nombre de marca, en España se usa en la lengua hablada otro nombremarca: cel·lo $m$ (pronunciado celo). 


\section{SUGERENCIAS PARA CORREGIR O MEJORAR LOS EQUIVALENTES DE LOS AMERICANISMOS REGISTRADOS}

\section{Peninsularismos}

Las unidades léxicas registradas son, en su inmensa mayoría, americanismos. Hemos encontrado tan sólo unos pocos peninsularismos, como: "chef $\mathrm{nm}$ "cocinero"' y "descolgarse 'aparecer', 'presentarse en un lugar"” (que tengo documentado para España desde 1958). "Operativo $\mathrm{nm}$ = operación (esp policial o militar) [Ven] usual en AmMer", que se registró también en el Nuevo Diccionario de Argentinismos (Haensch-Werner 1993 b) empieza a usarse en la prensa española y deja, por lo tanto, de ser americanismo. "Tipa $f[R P l]$ "tía" (con una cita del Uruguay). Tengo documentada la voz como usual en el lenguaje coloquial peninsular, junto a sus sinónimos tipeja e individua, desde los años sesenta.

\section{EXACTITUD DE LOS EQUIVALENTES Y OTRAS CORRECCIONES}

En algunos casos se podrían precisar o afinar más las definiciones o los equivalentes que, en vez de definiciones, sirven para indicar el significado de las palabras registradas.

"Aeroparque $m$ (Argentina) 'aeropuerto pequeño"' es, en realidad, un nombre propio y no debería figurar como nombre común. Existe sólo el Aeroparque de Buenos Aires, que es el aeropuerto para el tráfico nacional y con Uruguay, el aeropuerto internacional es el de Ezeiza; no hay otros aeroparques. Baba $f \mathrm{Col}$., Ven. y babilla $f \mathrm{Col}$. no corresponden a 'caimán' a secas (hay varias especies), sino al 'caimán de anteojos' (Caiman sclerops). "Botan(as) $n f(p l)$ 'tapa"'. El autor cita la obra de Angel Rosenblat: El castellano de España y el castellano de América, Madrid, Taurus 1970, p. 9 y da como marca geográfica para esta voz Venezuela, lo cual es un error. En Venezuela, las tapas se llaman pasapalos, como lo dice Steel correctamente en el artículo pasapalos, pero como el autor añade, además de la marca Ven la de Méx, hay otro error. Las 'tapas' se llaman en México botanas o antojitos, esta última palabra está mal definida en el diccionario como 'comida 
típica popular' (con la marca Méx). El texto de Rosenblat es algo ambiguo, pero en las páginas 8 y 9 el autor se refiere al ambiente mejicano y da sólo equivalentes de voces usuales en otros países hispanoamericanos entre paréntesis. "Cachaco $\mathrm{m}$ adj y $\mathrm{nm}$ fam $[\mathrm{Col}]=$ (persona) de Bogotá." Esta entrada necesita una ampliación. Cachaco $m$ es, en Colombia, en primer lugar, 'una persona bien educada, de buenos modales', luego, 'persona elegante y bien vestida'. Para los hablantes de la Costa atlántica (y no los otros colombianos) es un 'natural del interior de Colombia', especialmente del Altiplano (Cundinamarca y Boyacá) (datos del NDC), y, por lo tanto, también 'persona de Bogotá'. Como cachaco tiene un matiz despectivo, los costeños usan para designar a los habitantes del interior del país la voz interiorano. Me parece poco recomendable, como se hizo en este caso, utilizar como fuente para documentar un colombianismo, un periódico de Guadalajara, Méjico. Para "curita $f$ 'tira de vendaje plástico"" se da como equivalente peninsular tirita $f$, lo cual es correcto, pero habría que señalar que en ambos casos se trata de un "nombre-marca", es decir del nombre de un producto de una marca determinada empleado abusivamente como nombre genérico. "Potrero $\mathrm{m}$ nm fam", que Steel 1999 registra para Arg. con el equivalente 'campo', nos parece inexacto. Un potrero es una 'parcela de terreno cercado cubierto de hierba que sirve para el pastoreo del ganado bovino y de caballos', en España: 'dehesa'. En cambio, la traducción inglesa 'paddock' que da el autor es correcta. La voz se usa casi en toda Hispanoamérica. "Prender $v t$ " aparece con el significado 'encender' con la marca Méx en la primera parte de la obra y luego con el mismo equivalente 'encender' en la Parte II “Americanismos de frecuencia". "Pueblo $m$ joven $\mathrm{nm}[\mathrm{Pe}]=$ "barrio de chabolas"' no es la palabra normal que se usa en Perú para designar los 'barrios de chabolas'. Es un eufemismo administrativo y, a veces periodístico, algo cínico, para designar lo que los limeños llaman normalmente barriadas, chozas o ranchos. "Víbora de la cruz $n f$ (rioplatense $)=$ varias especies de serpiente; Bothrops y yarará $[$ Par $]$ 'serpiente muy venenosa; familia Bothrops"'. Ambas voces designan en Arg., Ur. y Par dos serpientes venenosas: Bothrops alternatus y Bothrops neuwiedii. El artículo "surubí $\mathrm{nm}$ (rioplatense) Pez de agua dulce, 
similar a un bagre [...]" se podría completar por más información de interés para los lectores. El surubí que tiene forma de siluro puede alcanzar 1,50 m de largo, su carne es muy apreciada (Familia Pimelodidae; géneros Sorubim y Pseudoplatystoma). En el artículo para "penetrar" leemos: "penetrar a $v i=$ penetrar en. En cayuco hay que penetrar al pantano. (M. Mejido [Méx.] 1984: 181) to go into." Mientras que "penetrar a" figura en la $1^{a}$ parte del diccionario; "entrar a" aparece en la $2^{a}$ parte "Americanismos de frecuencia". Todo esto es correcto, pero pensamos que hubiera sido mejor introducir en el capítulo "Otros americanismos morfológicos y sintácticos" un artículo sobre este uso de "a", puesto que en el español de América la mayoría de los verbos, transitivos, intransitivos y pronominales, que expresan un movimiento dentro de algo se construyen con a, en España, en cambio, con en: entrar a la casa, internarse a la selva, meterse a la cama, etc. y no limitarse a dos ejemplos. "Boleto $m$ " figura en el capítulo "Palabras de orígen latinoamericano que ahora son de uso general en el español". Aquí hay que diferenciar. "Boleto $m$ " significa en España sólo "Papeleta con que se participa en una rifa o sorteo"16. En Hispanoamérica, en cambio, se usa para designar "billetes de tren, autobus", en algunos países también "billete de avión", por eso, la voz debería figurar en la primera parte del diccionario. En este caso importa más el significado diferente que el significante común. "Comida $f$ "cena" que Steel 1999 da para Arg y Cu, se usa también en Chile, Bol, Pe, Col, Pan y CR. Sería interesante registrar también en el diccionario: almuerzo $m$ nombre usual de la 'comida del mediodía' en toda Hispanoamérica, mientras que esta voz es, en España, de estilo elevado (un ministro invita a un almuerzo, se usa en hoteles y restaurantes de categoría), lo usual en España es comida $f$.

16 Diccionario Salamanca, p. 203. 


\section{COMPLEMENTO DE INFORMACIÓN SOBRE EL LÉXICO REGISTRADO EN STEEL 1999}

No como crítica, sino más bien como homenaje al autor y complemento para los lectores ofrecemos a continuación, como ampliación de este artículo-reseña, una selección de voces registradas en Steel 1999, pero completando la lista de los países donde se usan. En algunos casos se indican también variantes de una palabra o se señalan usos peninsulares divergentes ${ }^{17}$.

Para ganar espacio, usaremos el esquema siguiente:

\begin{tabular}{|c|c|c|}
\hline $\begin{array}{l}\text { Americanismo registrado } \\
\text { en Steel (1999), con su } \\
\text { equivalente en español } \\
\text { peninsular }\end{array}$ & $\begin{array}{l}\text { Marca geográfica en } \\
\text { Steel (1999) }\end{array}$ & 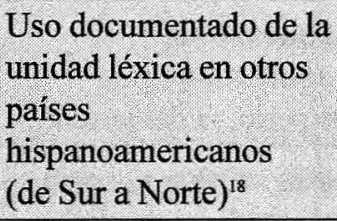 \\
\hline aeromoza $n f$ 'azafata' 19 & Chile & $\begin{array}{l}\text { Ur, Bol, Pe, Ec, Ven, } \\
\text { Pan, Nic, Guat, Salv }\end{array}$ \\
\hline afiche $m$ 'cartel' ${ }^{20}$ & $P e$ & $\begin{array}{l}\text { toda AmMer, CR, Guat, } \\
\text { Salv, RD, Cuba }\end{array}$ \\
\hline $\begin{array}{l}\text { almacen } m \text { 'tienda de } \\
\text { comestibles' }\end{array}$ & $R p l$ & Chile, Bol \\
\hline $\begin{array}{l}\text { antier 'anteayer' (la voz } \\
\text { es familiar en España) }\end{array}$ & Pe, Méx & $\begin{array}{l}\text { Bol, Col, Pan, Ven, } \\
\text { AmCent, RD, PR, Cuba }\end{array}$ \\
\hline $\begin{array}{l}\text { aplanadora } \mathrm{f} \\
\text { 'apisonadora' }\end{array}$ & Méx & $\begin{array}{l}\text { AmMer, CR, Nic, Guat, } \\
\text { Cuba }\end{array}$ \\
\hline
\end{tabular}

17 Para las siglas usadas, vid. nota 13.

18 Si no cito otra fuente, las voces (o sus marcas geográficas) están documentadas en una encuesta que realicé en todos los países hispanoamericanos en 1989 para reunir los equivalentes hispanoamericanos de 750 palabras muy usuales del español peninsular.

19 Según me comunica D. Manuel Seco, aeromoza, hoy raro en España, se ha usado allí entre 1951 y 1969.

20 La voz es de uso restringido en España. 


\begin{tabular}{|c|c|c|}
\hline $\begin{array}{l}\text { Americanismo registrado } \\
\text { en Steel (1999), con su } \\
\text { equivalente en español } \\
\text { peninsular }\end{array}$ & $\begin{array}{l}\text { Marca geográfica en } \\
\text { Steel (1999) }\end{array}$ & 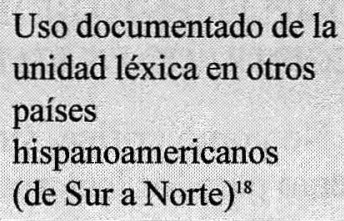 \\
\hline arveja $f$ 'guisante' & $\operatorname{Arg}$ & $\begin{array}{l}\text { AmMer, } C R, R D . \\
\text { La variante alverja se } \\
\text { usa en } U r, \text { Par, Bol, Pe, } \\
\text { Col, CR. Esta es la voz } \\
\text { usual en Guat y Salv }\end{array}$ \\
\hline atorarse 'atragantarse' & Méx, $P e$ & general en $\mathrm{Am}$ \\
\hline $\begin{array}{l}\text { baqueano,-a, } \\
\text { baquiano,-a 'guía' } \\
\text { (experto, -a del terreno) }\end{array}$ & Cuba & $\begin{array}{l}\text { RPl, Bol, Col, Ven, CR, } \\
\text { Nic }\end{array}$ \\
\hline $\begin{array}{l}\text { botar vt 'tirar } \mathrm{u} / \mathrm{c} \text { para } \\
\text { desprenderse de ella' }\end{array}$ & Chile & $\begin{array}{l}\text { Bol, Pe, Ec, Col, Ven, } \\
\text { AmCent, Méx, RD, PR, } \\
\text { Cuba }\end{array}$ \\
\hline cachetada $\mathrm{f}$ 'bofetada' & Méx & $\begin{array}{l}\text { toda } A m M e r, \text { toda } \\
\text { AmCent, RD }\end{array}$ \\
\hline $\begin{array}{l}\text { canciller } \mathrm{nm} \text { 'ministro } \\
\text { de Asuntos exteriores' }\end{array}$ & Méx, Ven & general en toda $\mathrm{Am}$ \\
\hline $\begin{array}{l}\text { carpa } f \text { 'tienda de } \\
\text { campaña' }\end{array}$ & $P e$ & $\begin{array}{l}\text { Arg, Ur, Par, Chile, Ec, } \\
\text { Col; se usa también, } \\
\text { junto a la voz tienda de } \\
\text { campaña, en Bol, Ven, } \\
\text { Guat, Salv }\end{array}$ \\
\hline 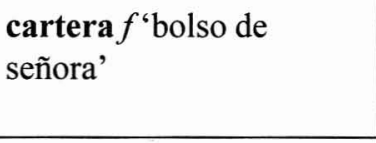 & Chile, $P e$ & $\begin{array}{l}\text { Arg, Par, Pe, Ec, Col, } \\
\text { Ven, Pan, Salv, PR, } \\
\text { Cuba, RD }\end{array}$ \\
\hline chancho $m$ 'cerdo' & $U r$ & Arg, Par, Chile, Pe \\
\hline
\end{tabular}

21 Botar debería figurar entre los americanismos de la $1^{\text {a }}$ parte. En España la voz usual es tirar. Botar es de uso regional en Mallorca, Canarias y Galicia. 


\begin{tabular}{|c|c|c|}
\hline $\begin{array}{l}\text { Americanismo registrado } \\
\text { en Steel (1999), con su } \\
\text { equivalente en español } \\
\text { peninsular }\end{array}$ & $\begin{array}{l}\text { Marca geográfica en } \\
\text { Steel (1999) }\end{array}$ & $\begin{array}{l}\text { Uso documentado de la } \\
\text { unidad léxica en otros } \\
\text { países } \\
\text { hispanoamericanos } \\
\text { (de Sur a Norte) }^{18}\end{array}$ \\
\hline $\begin{array}{l}\text { chapa } f \text { 'cerradura' } \\
\text { (de una puerta) }\end{array}$ & Chile & $\begin{array}{l}\text { Bol, Pe, Ec, Col, Hond, } \\
\text { Guat, Salv, Méx }\end{array}$ \\
\hline $\begin{array}{l}\text { chiflón } m \text { 'corriente de } \\
\text { aire' }\end{array}$ & Méx & $\begin{array}{l}\text { Arg, Chile, Bol, Pe, Col, } \\
\text { AmCent }\end{array}$ \\
\hline chinchorro $m$ 'hamaca' & Chile, Ven & Col, Pan \\
\hline $\begin{array}{l}\text { cloch }(\mathbf{e}) m \text {, clutch } \\
\text { 'embrague' }\end{array}$ & Méx & $\begin{array}{l}\text { cloche } m \text { en } V e n, R D, \\
C u b a, P R ; \text { cloch } m \text { en } \\
C o l, \text { toda } A m C e n t\end{array}$ \\
\hline $\begin{array}{l}\text { clóset } n m \text { 'armario } \\
\text { empotrado' }\end{array}$ & Cuba, Ven & $\begin{array}{l}\text { Chile, Bol, Pe, Ec, Col, } \\
\text { toda AmCent, Méx, RD }\end{array}$ \\
\hline $\begin{array}{l}\text { convertible } \mathrm{nm} \\
\text { 'automóvil descapotable' }\end{array}$ & Méx & $\begin{array}{l}\text { toda } A m M e r \text { y } A m C e n t, \\
R D, C u b a\end{array}$ \\
\hline coto $m$ 'bocio' & Chile & Arg, Par, Bol, Col, Nic \\
\hline 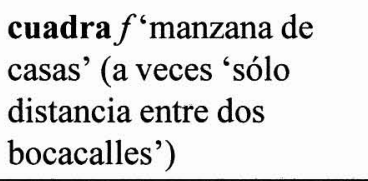 & Chile & general en $\mathrm{Am}$ \\
\hline $\begin{array}{l}\text { cuy } m \text { 'conejillo de } \\
\text { Indias' }\end{array}$ & Chile, Pe & $\begin{array}{l}\text { Arg, Ec, Pan, Guat; la } \\
\text { variante cuyo se usa en } \\
\text { Guat, Hond, Salv, Méx. } \\
\text { La variante cuis m se } \\
\text { usa en } U r \text { y Bol. }\end{array}$ \\
\hline $\begin{array}{l}\text { Desempeñarse como } \\
\text { 'trabajar de' }\end{array}$ & Ven & $\begin{array}{l}\text { Arg, Par, Chile, Bol, Pe, } \\
\text { CR, Nic, Guat, Salv, RD }\end{array}$ \\
\hline $\begin{array}{l}\text { encomienda } f \text { 'paquete' } \\
\text { (postal) }\end{array}$ & $\operatorname{Arg}$ & $\begin{array}{l}\text { general en AmMer y } \\
\text { AmCent }\end{array}$ \\
\hline $\begin{array}{l}\text { estadía } f \text { 'estancia', } \\
\text { 'permanencia' }\end{array}$ & $\operatorname{Arg}$ & $\begin{array}{l}\text { Ur, Par, Chile, Bol, Pe, } \\
\text { Ec, Col, Ven, CR, Nic, } \\
\text { Hond, Guat, Salv; Méx }\end{array}$ \\
\hline
\end{tabular}




\begin{tabular}{|c|c|c|}
\hline $\begin{array}{l}\text { Americanismo registrado } \\
\text { en Steel (1999), con su } \\
\text { equivalente en español } \\
\text { peninsular }\end{array}$ & $\begin{array}{l}\text { Marca geográfica en } \\
\text { Steel (1999) }\end{array}$ & $\begin{array}{l}\text { Uso documentado de la } \\
\text { unidad léxica en otros } \\
\text { países } \\
\text { hispanoamericanos } \\
\text { (de Sur a Norte) }^{18}\end{array}$ \\
\hline & & $\begin{array}{l}\text { (allí menos frecuente } \\
\text { que estancia); } R D\end{array}$ \\
\hline $\begin{array}{l}\text { estampilla } f \text { 'sello de } \\
\text { correos' }\end{array}$ & Arg & $\begin{array}{l}\text { AmMer, Pan, CR, Nic, } \\
\text { Hond, Salv }\end{array}$ \\
\hline frazada $f$ 'manta' & $\mathrm{Pe}$ & $\begin{array}{l}\text { Arg, Ur, Par, Chile, Bol, } \\
\text { Pe, Nic, Salv, Cuba }\end{array}$ \\
\hline guachimán $m$ 'vigilante' & Chile (junto a rondín $m$ ) & $\begin{array}{l}P e, C o l \text { (especialmente } \\
\text { en la Costa atlántica), } \\
\text { Ven, } C R, R D \text {, obsoleto } \\
\text { en Nic, } P R\end{array}$ \\
\hline $\begin{array}{l}\text { jalar } v t \text { 'tirar' ( } \mathrm{u} / \mathrm{c} \text { hacia } \\
\text { si) (de uso regional, en } \\
\text { Andalucía) }\end{array}$ & Méx & $\begin{array}{l}\text { Pe, Col, Ven, Pan, CR, } \\
\text { Nic, Hond, Guat, Salv, } \\
\text { Méx, RD, Cuba, PR }\end{array}$ \\
\hline mamadera $f^{\prime}$ 'biberón' & Chile & $\begin{array}{l}\text { Arg, Ur, Par, Bol, Pe, Ec, } \\
\text { Pan, Nic, Hond }\end{array}$ \\
\hline maní $m$ 'cacahuete' & Chile, Ec, Pe, Ur, Ven & $\begin{array}{l}\text { Arg, Par, Bol, Col, Ven, } \\
\text { Pan, CR, Nic, Hond, } \\
\text { Guat, RD, Cuba, PR }\end{array}$ \\
\hline $\begin{array}{l}\text { mayoreo } \mathrm{nm} \text { 'venta o } \\
\text { comercio al por mayor' }\end{array}$ & Méx & Chile, Bol, Pe, AmCent \\
\hline mesero $\mathrm{nm}$ 'camarero' & Col, Guat, Méx, Pe & Pan, CR, Nic, Salv \\
\hline $\operatorname{mozo} m$ 'camarero' & $U r$ & Arg, Par, Bol, Pe, Ec \\
\hline $\begin{array}{l}\text { nieve } f \text { 'helado de frutas, } \\
\text { aguas y azúcar' }\end{array}$ & Méx & Guat, Hond, Nic \\
\hline panqueque $m$ 'crepe' & Chile & $\begin{array}{l}\text { Arg, Ur, Par, Pe, Bol, } \\
\text { Col, Pan, CR, Nic, } \\
\text { Hond, Guat, Salv, PR }\end{array}$ \\
\hline $\begin{array}{l}\text { pararse 'levantarse', } \\
\text { 'ponerse en pie' }\end{array}$ & $\begin{array}{l}\text { sin marca geográfica en } \\
\text { Steel } 1999\end{array}$ & usual en toda Am \\
\hline
\end{tabular}




\begin{tabular}{|l|l|l|}
\hline $\begin{array}{l}\text { Americanismo registrado } \\
\text { en Steel (1999), con su } \\
\text { equivalente en español } \\
\text { peninsular }\end{array}$ & $\begin{array}{l}\text { Marca geográfica en } \\
\text { Steel (1999) }\end{array}$ & $\begin{array}{l}\text { Uso documentado de la } \\
\text { unidad léxica en otros } \\
\text { paises } \\
\text { hispanoamericanos } \\
\text { (de Sur a Norte) }\end{array}$ \\
\hline plata $n f$ 'dinero' & Ven & $\begin{array}{l}\text { usual en toda } \text { AmMer y } \\
\text { AmCent }\end{array}$ \\
\hline $\begin{array}{l}\text { recibirse de } \\
\text { 'licenciarse'22 (mejor, } \\
\text { por ser más general, } \\
\text { 'graduarse') }\end{array}$ & Arg, Méx & $\begin{array}{l}\text { Ur, Par, Chile, Bol, Pe, } \\
\text { Nic, Guat, Salv }\end{array}$ \\
\hline $\begin{array}{l}\text { saco } n m \text { 'chaqueta', } \\
\text { 'americana' }\end{array}$ & Méx & $\begin{array}{l}\text { Arg, Ur, Par, Bol, Pe, } \\
\text { Col, Ven, Pan, Nic, } \\
\text { Guat, Salv, Méx, RD, } \\
\text { Cuba }\end{array}$ \\
\hline $\begin{array}{l}\text { sindicar vt'inculpar' } \\
\text { (de un delito) }\end{array}$ & Bol, Ec, Rpl, Ven & $\begin{array}{l}\text { Arg, Par, Col, Pan, Nic, } \\
\text { Guat, Salv }\end{array}$ \\
\hline $\begin{array}{l}\text { suich/suiche } n m \\
\text { 'interruptor'; 'botón' }\end{array}$ & Col, Méx, PR, Ven & $\begin{array}{l}\text { suich en CR, Nic, Hond, } \\
\text { Guat, Salv; suiche en } \\
\text { Pan, CR, Salv, RD }\end{array}$ \\
\hline $\begin{array}{l}\text { taco } m \text { 'tacón del zapato' } \\
\text { (de una calle) }\end{array}$ & Chile, Pe, RPl & Bol, Ec \\
\hline vigrillo $m$ 'ocelote' $m$ 'volante & AmCent, Col, Ec, Méx, & Bol, Pe \\
\hline Ven & Méx & $\begin{array}{l}\text { Pe, Col, Pan, Nic, Hond, } \\
\text { Guat, Salv, RD, Cuba }\end{array}$ \\
\hline $\begin{array}{l}\text { verg, Ur, Par, Chile, Bol, } \\
\text { Ec }\end{array}$
\end{tabular}

22 La voz es de uso restringido en España.

23 La voz es de uso restringido en España. 


\begin{tabular}{|c|c|c|}
\hline $\begin{array}{l}\text { Americanismo registrado } \\
\text { en Steel (1999), con su } \\
\text { equivalente en español } \\
\text { peninsular }\end{array}$ & $\begin{array}{l}\text { Marca geográfica en } \\
\text { Steel (1999) }\end{array}$ & $\begin{array}{l}\text { Uso documentado de la } \\
\text { unidad léxica en otros } \\
\text { paises } \\
\text { hispanoamericanos } \\
\text { (de Sur a Norte) }^{18}\end{array}$ \\
\hline $\begin{array}{l}\text { yapa } f \text {, variante: ñapa } f \\
\text { 'pequeña cantidad que } \\
\text { añade un comerciante a } \\
\text { la compra' }\end{array}$ & $\begin{array}{l}\text { yapa } f \text { en } A r g, P e ; \\
\text { ñapa } f \text { en } A r g\end{array}$ & $\begin{array}{l}\text { yapa } f \text { en } U r, \text { Par, Chile, } \\
\text { Bol, Ec; ñapa } f \text { en Col, } \\
\text { Ven, Pan, } R D \text {. En Méx: } \\
\text { ñapa y yapa }\end{array}$ \\
\hline $\begin{array}{l}\text { zíper } n m \text { 'cremallera' } \\
\text { (de una prenda de vestir) }\end{array}$ & AmCent, Cuba, Méx & $\begin{array}{l}\text { con la variante cíper } \\
\text { usual en } R D, P R\end{array}$ \\
\hline
\end{tabular}

\section{CONCLUSIÓN}

Resumiendo los comentarios sobre Steel 1999 podemos afirmar que, a pesar de las limitaciones inherentes al método aplicado (que no son, insistimos en esto, deficiencias), ciertas inconsecuencias metodológicas y algunas inexactitudes que habría que corregir, la obra es una aportación útil a la lexicografía del español de América. Si decíamos antes que hasta cierto punto se trata de una 'cosecha del azar', podemos añadir al final: pero, ¡qué cosecha! Para conocer mejor el riquísimo léxico del español de América harán falta muchos diccionarios, nacionales y regionales, bien sean diferenciales-contrastivos, o bien sean integrales, así como glosarios de jergas, listas de gentilicios, terminologías técnicas, pero también repertorios lexicográficos basados en una documentación, como la obra comentada, que enriquece la documentación actualizada sobre el léxico del español de América. 


\section{BIBLIOGRAFÍA}

Americanismos (1983): Diccionario Ilustrado Sopena, Barcelona, pp. 670.

ARIAS DE LA CRUZ (1980): Diccionario temático de americanismos, León. CAUDMONT, Jean (1968): "La situation linguistique en Colombie", en André Martinet (ed.), Encyclopédie de la Pléiade. Le Langage, Paris, pp. 1188-1202.

Corominas, Juan/ PAScual, José Antonio (1980-1991): Diccionario critico etimológico castellano e hispánico, Madrid.

Diccionario Salamanca = GüTIÉRREZ CUADRADO, Juan (ed.) (1996): Diccionario Salamanca de la lengua española, Madrid y Salamanca.

Diccionario Sopena (1978): Diccionario Enciclopédico Ilustrado Sopena, Barcelona, pp. 4583 (sin los apéndices, éstos sin paginación).

GLESSGEN, Martin-Dietrich (1998): “Aspekte hispanoamerikanischer Lexikographie", Zeitschrift für romanische Philologie, 114 (1998), pp. 290-300.

Gran Sopena (1980): Gran Sopena. Diccionario Ilustrado de la Lengua Española, Barcelona, 3 ts.

HAENSCH, Günther (1978): "Zur Lexikographie des amerikanischen Spanisch. Heutiger Stand und Überblick über die Problematik", en Günther Haensch y Reinhold Werner (eds.): Referate der 1. wissenschaftlichen Tagung des Deutschen Hispanistenverbands Augsburg, 25.-26.6.1977, Augsburg, pp. 112-131.

— (1980): "Algunas consideraciones sobre la problemática de los diccionarios de español de América", Lingüística Española Actual, II, 2, 1980, pp. 375-384.

(1983): "Un nuevo Diccionario de Americanismos (NDA) y la problemática de la lexicografía del español de América", Anales del Instituto de Lingüistica de la Universidad Nacional de Cuyo (Mendoza), XI, pp. 111-117.

_ (1984 a): "Neues Wörterbuch des amerikanischen Spanisch und Neues Wörterbuch des kolumbianischen Spanisch", Hispanorama. Mitteilungen des Deutschen Spanischlehrerverbandes, 36, marzo de 1984, pp. 167-176.

—_ (1984 b): "Nuevo diccionario de americanismos. Neues Wörterbuch des amerikanischen Spanisch", en Wörterbücher der deutschen Romanistik, ed. por la Deutsche Forschungsgemeinschaft, Acta humaniora, Weinheim, pp. 93-104. 
(1984 c): "Miseria y esplendor de la lexicografía hispanoamericana", en Actas del VII Congreso de la Asociación de Lingüística y Filosofia de América Latina (ALFAL). Homenaje a Pedro Henríquez Ureña, t. I, Santo Domingo (R. D.), pp. 333-370.

— (1986): "La situación actual de la lexicografía del español de América", Revista de Filología Románica, Madrid, IV, pp. 281-293.

- (1987): "La lexicografía hispanoamericana entre la teoría y la práctica", en Actas del I Congreso Internacional sobre el Español de América, San Juan de Puerto Rico del 4 al 9 de octubre de 1982, San Juan, pp. 555-577.

_ (1988): "Antécédents et situation actuelle de la lexicographie de l'espagnol d'Amérique", Revue québécoise de linguistique, vol. 17, $\mathrm{n}^{\mathrm{o}} 2$, pp. 37-60.

_ (1989): "Der Wortschatz des amerikanischen Spanisch und seine Erfassung in lexikographischen Inventaren", Iberoromania, 30, Tubinga, pp. 1-25.

— (1991): "La lexicografía del español de América en el umbral del siglo XXI", en Presencia y destino. El español de América hacia el siglo XXI, t. I, Instituto Caro y Cuervo, Bogotá, pp. 41-77.

_ (1994): "Dos siglos de lexicografía del español de América: lo que se ha hecho y lo que queda por hacer", en Gerd Wotjak/ Klaus Zimmermann (eds.): Unidad y variación léxica del español de América, Frankfurt, pp. 39-81.

- (1997): Los diccionarios del español en el umbral del siglo XXI, Universidad de Salamanca, Salamanca.

HAENSCH, Günther/ WeRNER, Reinhold (1982 a): "Un nuevo diccionario de americanismos", Boletín de la Asociación Europea de Profesores de Español, XIV, 25, Madrid, pp. 102-105.

- (1982 b): "Noticias sobre un nuevo diccionario de americanismos", Yelmo, 52 y 53, Madrid, pp. 26-27.

— (1992): "Nuevo Diccionario de Americanismos. Un proyecto hispanoamericano con sede principal en Alemania", Diálogo Científico, vol. $1, \mathrm{n}^{\circ}$ 2, Tubinga, pp. 129-134.

— (eds.) (1993 a): Nuevo Diccionario de Colombianismos, Bogotá, Instituto Caro y Cuervo.

(eds.) (1993 b): Nuevo Diccionario de Argentinismos, redactado por Claudio Chuchuy y Laura Hlavacka, Bogotá, Instituto Caro y Cuervo. 
(eds.) (1993 c): Nuevo Diccionario de Uruguayismos, redactado por Ursula Kühl de Mones et al., Bogotá, Instituto Caro y Cuervo.

HEDIGER, Helga (1977): Particularidades léxicas en la novela hispanoamericana contemporánea, Bern.

LARA, Luis Fernando (ed.) (1982/1993): Diccionario fundamental del español de México, El Colegio de México, $2^{\mathrm{a}}$ ed.

- (1986): Diccionario básico del español de México, El Colegio de México.

- (1996): Diccionario del español usual en México, El colegio de México, pp. 941.

LÜDTKE, Jens (1997): "Nuevo diccionario de americanismos", Romanistisches Jahrbuch, 48, pp. 357-382.

Morales Pettorino, Félix/Quiroz Mejías, Óscar (1984-1987): Diccionario ejemplificado de chilenismos,

Morinigo, Marcos A. (1966): Diccionario manual de americanismos, $1^{\mathrm{a}}$ ed. Buenos Aires 1966, última reed. Anaya, Madrid 1993.

Neves, Alfredo (1973): Diccionario de americanismos, Buenos Aires, reimpr. 1975.

NúÑEZ, Rocío/PÉREZ, Francisco Javier (1994): Diccionario del habla culta de Venezuela. Venezolanismos, voces indígenas, nuevas acepciones, Universidad Católica Andrés Bello, Caracas.

Schwauss, María (ed.) (1977): Wörterbuch der regionalen Umgangssprache in Lateinamerika. Amerikanisch-Deutsch, Leipzig.

SECo, Manuel (1995): "Lexicografía del español en el fin de siglo", Donaire, 4, pp. 67-74.

TEJERA, María Josefina (1993): Diccionario de venezolanismos, 3 tomos, Caracas, Universidad Central.

WERNER, Reinhold (1978): "Zur Lexikographie des amerikanischen Spanisch. Vorschläge für ein neues Amerikanismenwörterbuch", en Günther Haensch y Reinhold Werner (eds.): Referate der 1. wissenschaftlichen Tagung des Deutschen Hispanistenverbands Augsburg 25.-26.2.1977, Augsburg, pp. 132-157.

(1979): "Zum Stand der Lexikographie des amerikanischen Spanisch", Ibero-Amerikanisches Archiv, Neue Folge 5, Berlín, pp. 121-160.

— (1984): "Die Amerikanismen im Handwörterbuch der Real Academia Española", en C. Segoviano/J. M. Navarro. Nuremberg (eds.): Spanien und Lateinamerika. Beiträge zu Sprache, Literatur, Kultur. Homenaje a Anton Bemmerlein, pp. 530-551. 
_ (1991): "Principios diferenciales y contrastivos en la lexicografía del español americano", en Encuentro internacional sobre el español de América. Presencia y destino. El español de América hacia el siglo XXI, t. I, Santafé de Bogotá, pp. 229-271.

- (1994): “¿Qué es un diccionario de americanismos?”, en Gerd Wotjak/Klaus Zimmermann (eds.): Unidad y variación léxicas del español de América, Frankfurt, pp. 9-38.

— (1996): "Neuere Wörterbücher des Spanischen in Amerika: Was ist neu an ihnen?", Lebende Sprachen, 3, pp. 98-112.

ZimmermanN, Klaus (1997): "Die Situation des Spanischen in Kolumbien", en W. Altmann, Th. Fischer, K. Zimmermann (eds.): Kolumbien heute; Politik. Wirtschaft. Kultur, Frankfurt/Main, pp. 393-416. 